\title{
Anquilose Estapedo-Vestibular: Estudo Retrospetivo de Cinco Casos em São Tomé e Príncipe
}

\section{Stapedo-Vestibular Ankylosis: Retrospective Study of Five Cases in São Tomé e Príncipe}

\author{
Paula CAMPELO $\rrbracket^{1}$, Cristina CAROÇA ${ }^{1,2}$, Catarina TINOCO ${ }^{1}$, Diogo OLIVEIRA E CARMO ${ }^{1,2}$, João PAÇO ${ }^{1,2}$
} Acta Med Port 2017 Oct;30(10):713-718 - https://doi.org/10.20344/amp.8568

\begin{abstract}
RESUMO
Introdução: A otosclerose é uma causa frequente de hipoacusia de condução caracterizada pela alteração da remodelação óssea localizada exclusivamente à cápsula ótica. Diferenças rácicas são evidentes na literatura e, ao contrário dos caucasianos, as descrições na população de origem africana são raras. Neste trabalho pretende-se reportar cinco casos observados, e cirurgicamente confirmados, de otosclerose em indivíduos de raça negra, em São Tomé e Príncipe.

Material e Métodos: Desde fevereiro de 2011, efetuam-se consultas e cirurgias de Otorrinolaringologia no Hospital Ayres de Menezes, em São Tomé e Príncipe, inseridas no projeto 'Saúde para todos - Especialidades'. Neste trabalho realizou-se um estudo retrospetivo dos doentes submetidos a estapedotomia ou estapedectomia parcial durante estas missões até fevereiro de 2014. Recolheu-se informação relativa à apresentação clínica, resultados audiométricos e relatórios cirúrgicos.

Resultados: Cinco doentes, adultos, foram submetidos a procedimento cirúrgico. Todos apresentavam otoscopia normal, ausência de história de traumatismo cranioencefálico ou quadro infecioso, audiograma com hipoacusia mista ou de condução e timpanograma tipo A, sem reflexos estapédicos. Em três doentes foi possível realizar audiograma pós-operatório, verificando-se melhoria do gap aero-ósseo.

Discussão: Neste trabalho documentam-se cinco casos de otosclerose clínica, e cirurgicamente confirmada, na população negra de São Tomé e Príncipe. Efetuada uma revisão temática, constata-se que, apesar de a otosclerose ser considerada rara nesta raça, os dados disponíveis parecem insuficientes para determinar se, de facto, diferentes raças têm diferentes incidências da doença.

Conclusão: Apesar de rara, a otosclerose não pode ser ignorada como uma possível causa de hipoacusia de condução em São Tomé e Príncipe.
\end{abstract}

Palavras-chave: África; Anquilose; Cirurgia do Estribo; Grupo com Ancestrais do Continente Africano; Otosclerose

\section{ABSTRACT}

Introduction: Otosclerosis is a common form of conductive hearing loss characterized by abnormal bone remodeling exclusively in the otic capsule. The prevalence of otosclerosis varies in racial populations and is described as being rare in black African populations. In this paper we aim to report five cases of clinical, and surgically confirmed, otosclerosis in black individuals, in São Tomé and Príncipe. Material and Methods: Since February 2011, Ear, Nose and Throat consultations and surgeries specialty have been carried out at Dr. Ayres de Menezes Hospital in cooperation with the project 'Health for all'. A retrospective analysis was undertaken of the records of all patients subjected either to stapedectomy or partial stapedectomy until February 2014. Information regarding clinical presentation, audiometric data and surgery reports was recorded.

Results: Five adult patients underwent stapedectomy or partial stapedectomy. All of them presented with normal otoscopy, conductive or mixed hearing loss on audiogram and normal tympanometry with absent stapedial reflexes. None of the patients had signs of infection or history of head trauma. Three cases showed improvement in the air-bone gap after surgery. The other two were lost to follow-up. Discussion: We documented and surgically confirmed five cases of clinical otosclerosis in this population. A thematic review was carried out and concluded that, despite being described as a rare event in this race, available literature on this topic is not enough to state that there is lower prevalence of otosclerosis amongst the African population.

Conclusion: Even if not common, otosclerosis cannot be disregarded as a possible cause for conductive hearing loss among the population of São Tomé and Principe.

Keywords: Africa; African Continental Ancestry Group; Ankylosis; Otosclerosis; Stapes Surgery

\section{INTRODUÇÃO}

A otosclerose é uma doença primária e exclusiva da cápsula ótica do ser humano. ${ }^{1}$ Esta patologia do metabolismo ósseo decorre da alteração do processo de remodelação ósseo numa estrutura que, em condições normais, tem pouca ou nenhuma atividade osteoclástica ou osteoblástica. ${ }^{1,2}$ Como consequência, ocorre uma ossificação da cápsula ótica e platina do estribo com a presença de vários focos de reabsorção e reposição óssea. Por norma, as lesões otoscleróticas iniciam-se na vizinhança das fissula ante fenestram e são limitadas ao tecido ósseo localizado anteriormente à janela oval que leva à calcificação do ligamento anular ou à fixação do estribo. São estas alterações que vão condicionar o desenvolvimento de uma surdez de condução. ${ }^{1,2}$ No entanto, estas lesões podem também afetar a cóclea e labirinto ósseo, com consequente surdez neurossensorial ou envolver tanto a cadeia ossicular como a cóclea com consequente surdez mista. ${ }^{2}$

Apesar dos inúmeros trabalhos de investigação desenvolvidos, a etiologia da otosclerose permanece por esclarecer. ${ }^{2,3}$ Atualmente considera-se a otosclerose como uma

\footnotetext{
1. Centro Clínico e Universitário de Otorrinolaringologia. Hospital CUF Infante Santo. Lisboa. Portugal.

2. Departamento de Otorrinolaringologia. NOVA Medical School. Faculdade de Ciências Médicas de Lisboa. Lisboa. Portugal.

$\triangle$ Autor correspondente: Paula Campelo. paulabcampelo@gmail.com
}

Recebido: 13 de dezembro de 2016 - Aceite: 04 de maio de 2017 | Copyright @ Ordem dos Médicos 2017 
doença multifatorial. A associação entre fatores genéticos e o meio ambiente parece ser a etiologia mais provável para o desenvolvimento desta entidade. ${ }^{3}$

Clinicamente, os doentes apresentam uma hipoacusia progressiva de condução, mista ou neurossensorial, que surge com frequência por volta da terceira à quinta década de vida. ${ }^{2}$ Parece clara na literatura a existência de uma predisposição racial na manifestação da otosclerose..$^{2-5} \mathrm{Em}$ estudos epidemiológicos, tanto a otosclerose clínica como a otosclerose histológica (detetada em exame histológico e independente da presença de sintomas clínicos) aparecem menos frequentemente na raça negra. ${ }^{2-5}$ No entanto, após revisão bibliográfica, encontram-se poucos trabalhos sobre a otosclerose em África, ${ }^{7}$ sendo este, tanto quanto é do conhecimento dos autores, o primeiro estudo sobre a otosclerose em São Tomé e Príncipe (STP).

As ilhas da atual República Democrática de São Tomé e Príncipe, foram descobertas e inicialmente povoadas por Portugueses, servindo como entreposto de escravos oriundos da Guiné; só posteriormente foram colonizadas por comerciantes, fidalgos, degradados e crianças judias, estas últimas provenientes de Espanha. ${ }^{8,9}$ Ao longo dos anos foram sendo invadidas por Angolanos, Ingleses, Franceses e Holandeses, tendo sido novamente integradas na Coroa Portuguesa em 1750, até à Independência em 1975. Atualmente STP tem cerca de 187000 habitantes, com uma população maioritariamente jovem. ${ }^{8-10}$

Desde fevereiro de 2011 que o Serviço de Otorrinolaringologia (ORL) do Hospital CUF Infante Santo tem participado no Projeto 'Saúde para todos - Especialidades' do Instituto Marquês de Valle Flor, com a realização de três missões por ano a esse país, com a duração de uma semana cada. Neste contexto realizaram-se consultas de ORL e exames de audiologia no Hospital Ayres de Menezes, localizado na ilha de São Tomé.

Na realização destas missões, os autores depararam-se com vários casos de otosclerose clínica na raça negra. Perante a ausência de artigos publicados sobre o tema nos últimos anos, e a crença geral da raridade da otosclerose nesta raça, pretende-se, com este trabalho, documentar os casos detetados de fixação da platina do estribo, cirurgicamente confirmados, na população negra de STP.

\section{MATERIAL E MÉTODOS}

Neste trabalho foi realizado um estudo retrospetivo com revisão da casuística cirúrgica, de fevereiro de 2011 a fevereiro de 2014, realizada pelas Equipas do Hospital CUF Infante Santo no Hospital Ayres de Menezes, em STP. Para o estudo foram incluídos apenas os doentes submetidos a cirurgia de otosclerose (estapedotomia ou estapedectomia) e estudadas as seguintes variáveis clínicas: género, idade à data da cirurgia, ouvido operado e história familiar de surdez. Através dos exames de audiologia realizados no pré- e pós-operatório, foi recolhida informação audiométrica relativa a estes doentes (gap aero-ósseo (GAO), limiares auditivos, tipo de timpanograma e presença de reflexos estapédicos ipsilaterais). Todos os doentes operados foram previamente avaliados pela equipa em consulta de ORL, onde realizaram audiograma tonal e impedanciometria (timpanograma com medição de reflexos ipsilaterais). Os exames audiométricos foram realizados por um audiologista. O equipamento utilizado foi o audiómetro Madsen Midimate 622. Devido à falta de recursos do Hospital local, os exames audiológicos foram realizados sem cabine audiométrica, com auscultadores TDH 39, em sala fechada, o que pode representar um viés de medição do estudo. No entanto, foram realizados com nível de ruído inferior a 35 dB medido pela aplicação iPhone de SchabelDoes/T GbR, Munich, Germany (versão 1.0.0) e considerado aceitável pela British Society of Audiology segundo o BS EN ISO 8253-1:1998..$^{11} \mathrm{O}$ equipamento de audiometria foi calibrado de acordo com as normas de calibração da BS EN ISO 389-3:1999. ${ }^{11}$

Audiologicamente, os doentes foram classificados segundo os critérios da Organização Mundial de Saúde, ${ }^{12}$ que considera o limiar de audição como a média aritmética dos limiares tonais nas frequências 0,5, 1, 2 e $4 \mathrm{kHz}$. Calculou-se o GAO, pré e pós-operatório, pela subtração do valor do limiar tonal médio da via aérea ao valor do limiar tonal médio da via óssea.

As cirurgias decorreram durante a presença das várias missões Portuguesas no Hospital Ayres de Menezes, tendo sido realizadas por diferentes cirurgiões.

\section{RESULTADOS}

Ao longo das missões foram observados um total de 573 doentes na consulta de Audiologia, dos quais 261 apresentavam critérios de surdez. Nas 229 cirurgias efetuadas (32 do foro otológico) detetou-se um total de cinco casos de doentes submetidos a cirurgia da otosclerose, após verificação intra-operatória da fixação da platina do estribo. Todos os doentes foram submetidos a cirurgia ao ouvido direito: três a estapedotomia e dois a estapedectomia parcial com colocação de pistão de teflon $\left(0,5 \mathrm{~mm}{ }^{*} 4,5 \mathrm{~mm}\right)$. Todos os doentes tiveram alta do internamento às 48 horas. Dois doentes apresentaram vertigem pós-operatória.

Os doentes operados por otosclerose perfazem $0,9 \%$ dos doentes observados na consulta, $1,9 \%$ dos doentes com hipoacusia e 2,2\% dos doentes operados durante as missões em STP (Tabela 1). Todos os doentes incluídos eram de raça negra e apresentavam, no pré-operatório, hipoacusia progressiva de condução, otoscopia sem alterações de relevo e ausência de história de traumatismo cranioencefálico ou infeção recente. Todos os casos

Tabela 1 - Número total de doentes observados durante o período em estudo

\begin{tabular}{lccc} 
& Doentes observados & Doentes com hipoacusia & Doentes com otosclerose clínica \\
\hline Consulta Audiologia & 573 & 261 & 5 \\
Bloco operatório & 229 & & 5 \\
\hline
\end{tabular}


Tabela 2 - Dados demográficos da população em estudo

\begin{tabular}{cccc}
\hline Caso & Idade & Género & Ouvido operado \\
\hline C1 & 23 & + & OD \\
C2 & 45 & $\delta$ & OD \\
C3 & 23 & $\delta$ & OD \\
C4 & 26 & $\delta$ & OD \\
C5 & 32 & $\delta$ & OD \\
\hline
\end{tabular}

negaram história de surdez na família.

Desses doentes operados, quatro eram do género masculino. A idade, à data da cirurgia, variou entre os $23 \mathrm{e}$ os 45 anos (Tabela 2). Os resultados audiométricos pré e pós-operatórios encontram-se resumidos na Tabela 3 . $\mathrm{Na}$ impedanciometria todos os doentes tinham timpanograma do tipo A sem reflexos estapédicos ipsilaterais, sendo que dois deles apresentavam timpanograma do tipo As (Fig. 1). Todos os casos apresentavam doença bilateral com GAO entre os 20 e os $63 \mathrm{~dB}$ (GAO médio de $37,8 \mathrm{~dB}$ ). Três casos apresentavam hipoacusia moderada e, os outros dois, hipoacusia severa pela classificação da Organização Mundial de Saúde. ${ }^{12}$

No pós-operatório, foi possível observar três doentes cujo GAO variou entre os $7 \mathrm{~dB}$ e os $15 \mathrm{~dB}$ com um GAO médio de $9,7 \mathrm{~dB}$, resultando num ganho médio de $28,1 \mathrm{~dB}$. Os outros dois casos foram perdidos para follow-up.

\section{DISCUSSÃO}

A otosclerose é a causa mais frequente da apresentação progressiva da surdez de condução nos adultos. ${ }^{1} \mathrm{Na}$ generalidade, a literatura aponta para uma clara predisposição racial no desenvolvimento da otosclerose.$^{1-5} \mathrm{~A}$ prevalência da otosclerose clínica, ou seja, sintomática, está estimada entre os $0,04 \%$ e $1 \%$ (média $0,3 \%$ ) nos caucasianos, ${ }^{6,13}$ enquanto nos asiáticos a prevalência é mais baixa ${ }^{14} \mathrm{e}$, na raça negra, praticamente nula, com algumas exceções já descritas. ${ }^{7,15}$ Por outro lado, a otosclerose histológica é considerada mais frequente, alcançando prevalências de $10 \%$ nos caucasianos, $5 \%$ nos asiáticos e $1 \%$ nos africanos, em estudos realizados nos Estados Unidos. ${ }^{5,16}$

$\mathrm{Na}$ última década foram poucos os artigos publicados
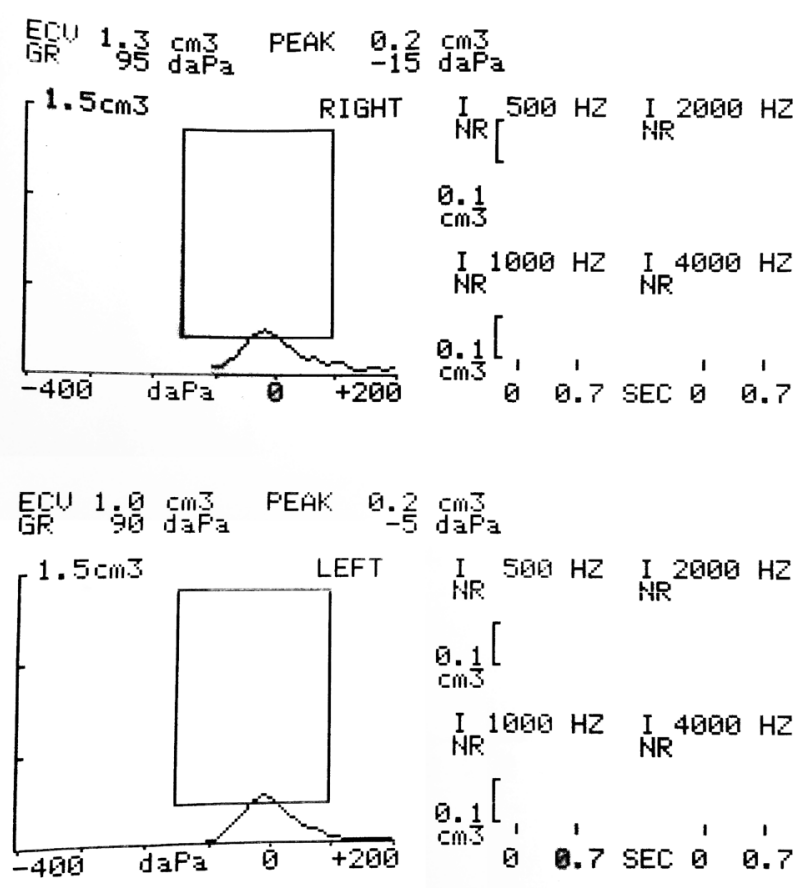

Figura 1 - Exemplo de impedanciometria: timpanograma tipo As, com ausência de reflexos estapédicos ipsilaterais

sobre a epidemiologia da otosclerose (Tabela 4). Os estudos sobre a raça negra, em particular na população Africana, são raros e, tanto quanto é do conhecimento dos autores, inexistentes na população de São Tomé e Príncipe. Em África, Rosen et al não detectaram casos no Sudão, ${ }^{2}$ enquanto Tshifularo et al documentaram 31 casos de otosclerose numa população negra indígena da África do Sul, onde a doença é considerada extremamente rara ou não existente. $^{7}$

No presente trabalho, verificou-se, em cinco casos, a existência intra-operatória de fixação do estribo, compatível clinicamente com o diagnóstico de otosclerose. Nos doentes com suspeita clínica de otosclerose a opção tem passado pelo tratamento cirúrgico, tanto pela eficácia já demonstrada na literatura, com baixa morbilidade, ${ }^{1}$ como pela dificuldade inerente em STP quanto à disponibilidade

Tabela 3 - Resumo dos resultados audiométricos pré e pós-operatórios

\begin{tabular}{|c|c|c|c|c|c|}
\hline Caso & Hipoacusia & Limiar tonal & Timpanograma & GAO pré-op & $\begin{array}{c}\text { GAO pós-op } \\
\text { (follow-up) }\end{array}$ \\
\hline \multirow{2}{*}{ C1 } & ODt: C & Moderada & As & 32 & 15 (9 meses) \\
\hline & OEsq: C & Moderada & As & 37 & \\
\hline \multirow{2}{*}{$\mathrm{C2}$} & ODt: M & Severa & A & 63 & 7 (12 meses) \\
\hline & OEsq: C & Moderada & A & 38 & \\
\hline \multirow{2}{*}{ C3 } & ODt: M & Moderada & $A$ & 48 & PF \\
\hline & OEsq: M & Moderada & $A$ & 42 & \\
\hline \multirow{2}{*}{$\mathrm{C} 4$} & ODt: M & Moderada & As & 33 & 7 (3 meses) \\
\hline & OEsq: M & Moderada & As & 23 & \\
\hline \multirow{2}{*}{ C5 } & ODt: M & Severa & A & 32 & PF \\
\hline & OEsq: M & Severa & A & 30 & \\
\hline
\end{tabular}

ODt: ouvido direito; OEsq: ouvido esquerdo: C: hipoacusia de condução; M: hipoacusia mista; GAO: gap aero-ósseo; PF: perdidos para follow-up 
Tabela 4 - Resumo dos dados da literatura sobre a otosclerose na população de origem africana em comparação com outras populações

\begin{tabular}{|c|c|c|c|c|c|c|}
\hline Autor & $\begin{array}{c}\text { Casos } \\
\text { (n) }\end{array}$ & Frequência relativa & $\begin{array}{c}\text { Idade } \\
\text { (M, anos) }\end{array}$ & $\begin{array}{c}\text { Género } \\
(1+1)\end{array}$ & $\begin{array}{c}\text { Lateralidade } \\
\text { (Bil:Unil) }\end{array}$ & $\begin{array}{l}\text { GAO } \\
\text { (M) }\end{array}$ \\
\hline \multicolumn{7}{|l|}{ População de raça negra } \\
\hline $\begin{array}{l}\text { População em estudo } \\
\text { (STP) }\end{array}$ & 5 & $\begin{array}{l}1,9 \%(\mathrm{DH}) \\
0,9 \%(\mathrm{DC})\end{array}$ & 29,8 & $4: 1$ & 5 & 37,8 \\
\hline $\begin{array}{l}\text { Tshifularo et al } \\
\text { (África do Sul) }\end{array}$ & 31 & $?$ & 41 & $9: 22$ & $27: 4$ & 40,3 \\
\hline $\begin{array}{l}\text { Seltzer }{ }^{15} \\
\text { (Filadélfia) }\end{array}$ & 16 & $?$ & $?$ & $\hat{\delta}<q$ & $?$ & $?$ \\
\hline \multicolumn{7}{|l|}{ Outras raças } \\
\hline $\begin{array}{l}\text { Sakihara et a/13 } \\
\text { (Caucasianos, Copenhaga) }\end{array}$ & 556 & $0,1 \%$ população & 75 & $166: 390$ & $?$ & $?$ \\
\hline $\begin{array}{l}\text { Yagii } \\
\text { (Asiáticos, Japão) }\end{array}$ & 80 & $0,22 \%(D C)$ & $?$ & $?$ & $72: 8$ & 31,7 \\
\hline
\end{tabular}

A frequência relativa é dada em relação à população total, ao número de doentes com hipoacusia (DH) ou ao número de doentes da consulta (DC). N: número total; M: média; Bil: bilateral; Unil: unilateral; GAO: gap aero-ósseo; ?: desconhecido

e acompanhamento na reabilitação protésica.

Os doentes operados apresentavam idades entre os 23 e os 45 anos, compatível com a idade descrita para o aparecimento de sintomatologia (entre os 30 e os 40 anos). Todos os casos apresentavam doença bilateral, como é frequente na otosclerose, onde a bilateralidade está presente em $70 \%$ a $80 \%$ dos doentes. ${ }^{5}$ Apesar da conhecida base hereditária na otosclerose, nesta amostra, todos os doentes negaram casos de surdez na família. Durante a realização das missões foi notória a diminuta literacia para a saúde desta população, nomeadamente a nível da importância e valorização da surdez. Este facto, por si só, pode justificar a ausência de relato de história familiar. Outros autores valorizaram já este efeito, salientando que, em várias séries, a história de surdez na família era apenas reportada em $49 \%$ - $58 \%$ dos casos, abaixo dos $70 \%$ de casos descritos como sendo de base hereditária. ${ }^{1}$

Dos 10 ouvidos estudados, cinco apresentavam surdez de condução e os outros cinco, uma surdez mista, todos com GAO superiores a $15 \mathrm{~dB}$ nas frequências de 500,1000 e $2000 \mathrm{~Hz}$. Num caso, é mesmo possível observar a incisura de Cahart (Fig. 2), caraterística audiológica típica da otosclerose. Nos dois doentes avaliados no pós-operatório é notória a redução do efeito de Cahart e encerramento do GAO (Fig. 3).

$\mathrm{Na}$ impedanciometria todos os doentes apresentavam uma compliance tipo $\mathrm{A}$, sendo que, em dois doentes, era do tipo As, típico da otosclerose mais avançada. Os reflexos estapédicos ipsilaterais são uma medida sensível ao movimento do estribo em reação à estimulação sonora ipsilateral. ${ }^{1} \mathrm{Na}$ otosclerose unilateral, devido à fixação da platina do estribo, estes estão caracteristicamente alterados (reflexo difásico) ou mesmo ausentes, mantendo-se o reflexo contralateral. ${ }^{1} \mathrm{Na}$ amostra em estudo nenhum doente apresentava reflexos estapédicos ipsilaterais. No entanto, sendo a doença bilateral em todos os casos e na ausência de informação sobre os reflexos contralaterais (o impedanciómetro utilizado não permite a medição destes reflexos), perante uma hipoacusia de condução não é possível concluir se a ausência de reflexos se deve a uma perda auditiva grave (via aferente do reflexo) ou à fixação da platina do estribo (via eferente) típica da otosclerose.

Em relação ao estudo imagiológico por tomografia computorizada, este não foi possível realizar uma vez que não

\section{AUDIOGRAMA TONAL}

\section{OUVIDO DIREITO}

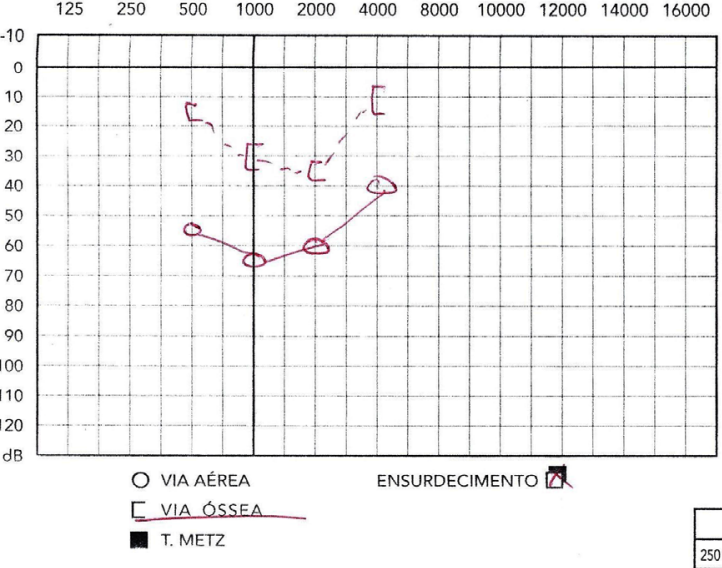

OUVIDO ESOUERDO

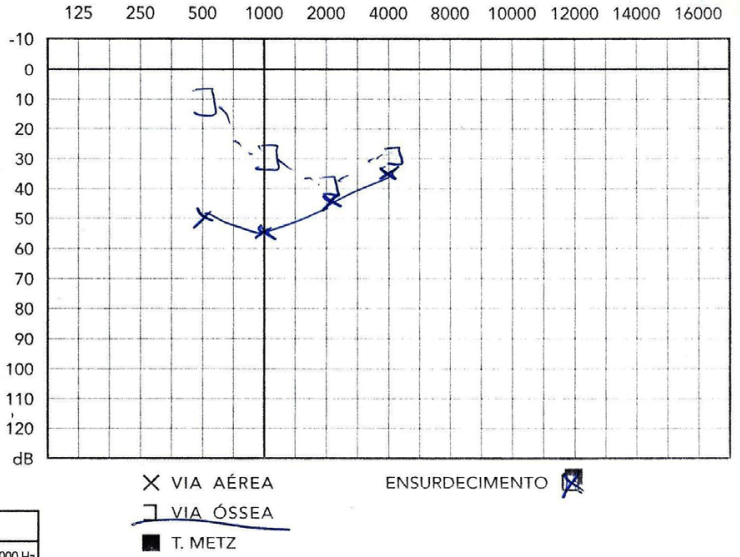

Figura 2 - Exemplo de audiograma pré-operatório: observa-se o efeito Cahart nos dois ouvidos 
OUVIDO DIREITO

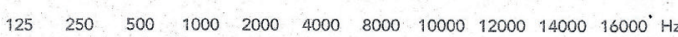

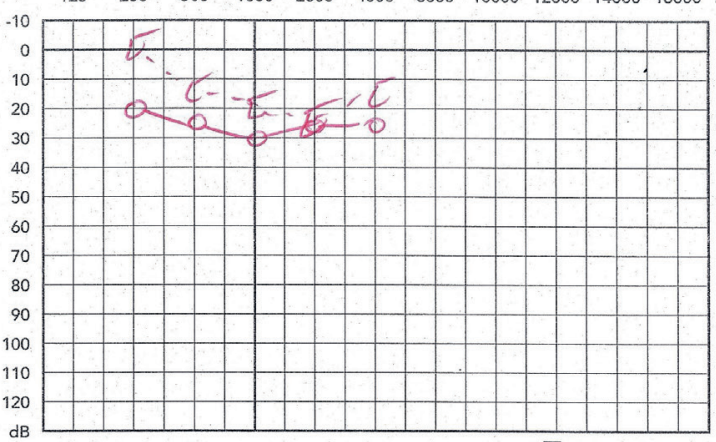

O VIA AÉREA

[ VIA ÓSSEA

T. METZ
ENSURDECIMENTO

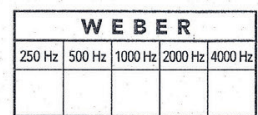

OUYIDO ESQUERDO

$\begin{array}{llllllllllll}125 & 250 & 500 & 1000 & 2000 & 4000 & 8000 & 10000 & 12000 & 14000 & 16000 & \mathrm{H} .\end{array}$ elap.

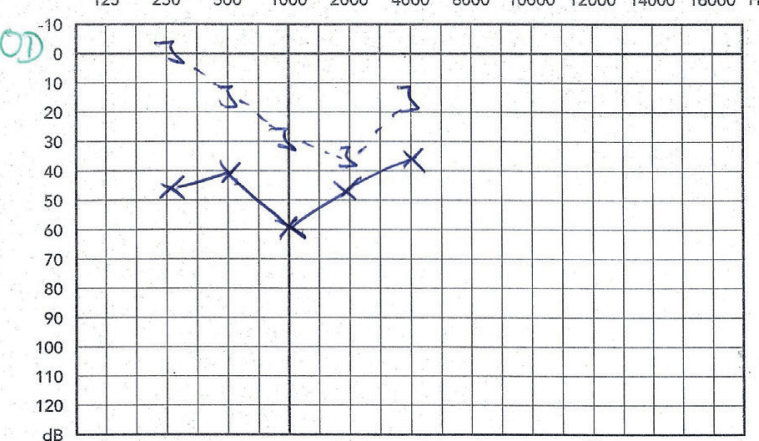

$\times$ VIA AÉREA

$\sqsupset$ VIA OSSEA

ENSURDECIMENTO

G. METZ

Figura 3 - Exemplo de audiograma pós-operatório: o efeito Cahart desaparece após encerramento do gap aero-ósseo à direita

está disponível em STP. Apesar da realização deste exame de imagem poder ser útil na confirmação do diagnóstico, extensão da doença e planeamento cirúrgico, sabe-se que as alterações tipicamente associadas à otosclerose não são específicas desta entidade. A utilização da tomografia computorizada no diagnóstico da otosclerose continua bastante controversa. ${ }^{1,17}$

Apesar de clinicamente os casos serem compatíveis com otosclerose, uma das limitações do trabalho é a impossibilidade de confirmar o diagnóstico através da realização de um estudo histológico. A fixação do estribo pode também estar associada a patologias como timpanosclerose, fixação congénita da platina do estribo, doença degenerativa, doença de Paget e osteogenesis imperfecta. ${ }^{1}$ No entanto, nenhum dos casos documentados apresentava outras alterações clínicas sugestivas destes diagnósticos diferenciais.

Perante a raridade da descrição da otosclerose na raça negra, torna-se importante documentar estes casos de fixação da platina do estribo na população negra de STP. As diferenças na manifestação da doença consoante a raça podem ser um reflexo de diferenças genéticas, não se devendo, contudo, esquecer eventuais diferenças ambientais. São várias as questões que se colocam. Desde logo, questiona-se se será a otosclerose praticamente inexistente nos africanos ou se estará apenas sub-diagnosticada pela ausência de estudos. Num artigo de opinião de 1961, Speltzer, Otorrinolaringologista de Filadélfia, defendia que a proporção, nos seus doentes, da otosclerose na raça negra versus popolação de origem caucasiana, não era tão diferente como se especulava e que fatores socioeconómicos poderiam justificar o sub-diagnóstico nos primeiros. ${ }^{15}$

Neste trabalho, os cinco casos detetados correspondem a $0,9 \%$ da população observada em consulta, um valor comparável à prevalência da otosclerose na população geral descrita nos caucasianos. Quando se compara a percentagem de otosclerose nos doentes com hipoacusia, o valor $(1,9 \%)$ é semelhante aos descritos em estudos asiáticos $(1,1 \%)^{18}$, onde a doença é considerada menos frequente $^{1,14}$ mas inferior aos dos caucasianos (5\%). ${ }^{1}$ Apesar da pequena amostra, estes dados indicam que a otosclerose apesar de pouco frequente na raça negra de STP, não é tão rara como se estaria à espera pela literatura.

Atualmente, a otosclerose é tida como uma doença multifatorial, causada pela associação de fatores genéticos (doença autossómica dominante com baixa penetração) com fatores ambientais (infeção por sarampo, fatores endócrinos ou baixas concentrações de fluoreto de sódio nas águas potáveis, por exemplo). ${ }^{4}$

Tendo por base estudos Japoneses, ${ }^{19}$ e a confirmar-se uma incidência mais elevada da otosclerose em STP, esta pode ser atribuída a: uma maior prevalência da otosclerose na ilha, uma maior atividade nos focos de otosclerose ou a diferentes localizações destes focos na cápsula ótica que cursem com maior repercussão clínica. Mais estudos epidemiológicos e histológicos serão necessários para confirmar estes dados.

Tendo em conta a história de STP, a própria colonização e as invasões por diferentes povos de raça caucasiana, ${ }^{9,10}$ pode-se, por outro lado, estar perante uma mistura rácica, o que poderia justificar o aparecimento dos casos descritos de otosclerose. Fatores ambientais, como por exemplo a infeção por sarampo, para a qual não existe vacinação no país, poderão contribuir, igualmente, para a manifestação da doença. Serão necessários, no futuro, mais estudos para se compreender a real prevalência e etiologia da otosclerose em STP.

\section{CONCLUSÃO}

Apesar da incidência e prevalência da otosclerose não serem ainda totalmente conhecidas em STP, esta doença deve ser tida em conta no diagnóstico diferencial da hipoacusia de condução nesta população.

A compreensão das diferenças raciais na manifestação clínica da otosclerose poderá contribuir para uma melhor clarificação da etiologia e prevenção da doença. 


\section{OBSERVAÇÕES}

Este trabalho foi apresentado em comunicação oral no $61^{\circ}$ Congresso Nacional da Sociedade Portuguesa de Otorrinolaringologia e Cirurgia Cervico-Facial, que se realizou entre 1 a 4 de maio de 2014, em Lisboa.

\section{PROTEÇÃO DE PESSOAS E ANIMAIS}

Os autores declaram que os procedimentos seguidos estavam de acordo com os regulamentos estabelecidos pelos responsáveis da Comissão de Investigação Clínica e Ética e de acordo com a Declaração de Helsínquia da Associação Médica Mundial.

\section{REFERÊNCIAS}

1. House J, Cunningham III C. Otosclerosis. In: Flint P, Haughey B, Lund V, Niparko J, Robbins K, Thomas J, et al, editors. Cummings otolaryngology: head and neck surgery. $6^{\text {th }}$ ed. Philadelphia: Saunders; 2015. p. 2211-9.

2. De Souza C, Glasscock III ME. Otosclerosis and stapedectomy: diagnosis, management \& complications. New York: Thieme; 2004. p. 3-22.

3. Karosi T, Sziklai I. Etiopathogenesis of otosclerosis. Eur Arch Otorhinolaryngol. 2010;267:1337-49.

4. Schrauwen I, Van Camp G. The etiology of otosclerosis: a combination of genes and environment. Laryngoscope. 2010;120:1195-202.

5. Markou K, Goudakos J. An overview of the etiology of otosclerosis. Eur Arch Otorhinolaryngol. 2009;266:25-35.

6. Declau F, Spaendonck M Van, Timmermans JP, Michaels L, Liang J, Qiu JP, et al. Prevalence of otosclerosis in an unselected series of temporal bones. Otol Neurotol. 2001;22:596-602.

7. Tshifularo M, Joseph C. Otosclerosis and TGF- $\beta 1$ gene in black South Africans. S Afr Med J. 2008;98:720-3.

8. Araujo MB. A ilha de São Tomé - alguns problemas históricos. Rev Univ Coimbra. 1991;36:261-76.

9. Tomas G, Seco L, Seixas S, Faustino P, Lavinha J, Rocha J. The peopling of Sao Tome (Gulf of Guinea): origins of slave settlers and admixture with the Portuguese. Hum Biol. 2002;74:397-411.

10. Trovoada MJ, Tavares L, Gusmão L, Alves C, Abade A, Amorim A, et al. Dissecting the genetic history of São Tomé e Príncipe: a new window from Y-chromosome biallelic markers. Ann Hum Genet. 2007;71:77-85.

\section{CONFIDENCIALIDADE DOS DADOS}

Os autores declaram ter seguido os protocolos do seu centro de trabalho acerca da publicação de dados.

\section{CONFLITOS DE INTERESSE}

Os autores declaram não existir qualquer conflito de interesse relativamente ao presente artigo.

\section{FONTES DE FINANCIAMENTO}

Este trabalho não foi subsidiado por quaisquer fundos provenientes de entidades no domínio público ou privado.

11. British Society of Audiology. Recommended procedure. Pure-tone air-conduction and bone-conduction threshold audiometry with and without masking [Internet]. 2011. [consultado 2017 mar 20]. Disponivel em: http://www.thebsa.org.uk/wp-content/uploads/2014/04/BSA VRA_24June2014_Final.pdf.

12. WHO. Guidelines for hearing aids and services for developing countries [Internet]. $2^{\text {nd }}$ ed. World Health Organization. Geneva; 2004 [consultado 2015 FeV 15]. Disponível em: http://www.who.int/pbd/deafness/en/ hearing_aid_guide_en.pdf.

13. Sakihara Y, Parving A. Clinical otosclerosis, prevalence estimates and spontaneous progress. Acta Otolaryngol. 1999;119:468-72.

14. Yagi T. Incidence and characteristics of otosclerosis in the Japanese population. Auris Nasus Larynx. 2002;29:257-60.

15. Seltzer A. The incidence of otosclerosis among negroes. J Natl Med Assoc. 1961;53:502-3.

16. Hueb MM, Goycoolea MV, Paparella MM, Oliveira JA. Otosclerosis: the University of Minnesota temporal bone collection. Otolaryngol Head Neck Surg. 1991;105:396-405.

17. Velegrakis GA. Otosclerosis: state of the art. Otorhinolaryngol Head Neck Surg. 2011:6-16.

18. Horiguchi S, Onchi Y. Studies on clinical otosclerosis in Japan. J Laryngol Otol. 1955;69:437-56.

19. Ohtani I, Baba Y, Suzuki T, Suzuki C, Kano M, Deka RC. Why is otosclerosis of low prevalence in Japanese? Otol Neurotol. 2003;24:377-81. 